\title{
Analytical Study for Chlorpyrifos Breakdown Derivatives Qualification After Silver Vanadate (Agvo3) Nanoparticles Fragmentation Reaction
}

Islam R. Ghoniem ( $\sim$ islam_refaat@qcap-egypt.com )

Agricultural Research Center https://orcid.org/0000-0001-6643-263X

Ashraf M. Elmarsafy

Agricultural Research Center

Mohamed A. Amer

Agricultural Research Center

Hassan A. El- Gammal

Agricultural Research Center

Mohamed M. Abo Aly

Ain Shams University Faculty of Science

Mostafa A. Sayed

Ain Shams University Faculty of Science

\section{Research Article}

Keywords: chlorpyrifos, silver vanadate, nanoparticles, LC-MS/MS, dimethoate, malathion

Posted Date: September 15th, 2021

DOl: https://doi.org/10.21203/rs.3.rs-653522/v1

License: (9) (i) This work is licensed under a Creative Commons Attribution 4.0 International License.

Read Full License 


\section{Abstract}

The use of pesticides in agriculture has become one of the basic necessities to meet the world's food requirements as a result of the continuous population increase. But sometimes the use of some of these pesticides in large quantities and in high concentrations poses a great danger to the environment and then to humans. Through weather factors, pesticides are transferred to the water and then harm aquatic organisms, which leads to a direct impact and harm to the fish wealth. Monitoring these compounds in the environment requires a large cost of time and money to ensure food safety. Therefore, a new method was used in this study in an attempt to get rid of these compounds in water. The silver vanadate nanoparticles (AgV03) were synthesized using a novel method in an attempt to eliminate different groups of pesticides that had been detected in Egyptian agricultural crops and water. The effect of $0.1 \mathrm{~g}$ of AgVO3 nanoparticles were found on three compounds: chlorpyrifos, dimethoate and malathion. Where a concentration of these compounds, which is $0.5 \mathrm{mg} / \mathrm{kg}$, was used and it was estimated using Exion HPLC coupled with Sciex Qtrap API 6500 + LC-MS/MS System. After 15 minutes of treatment with $0.1 \mathrm{~g}$ of AgVO3 nanoparticles, these compounds were undetectable on the device's chromatogram, which is regarded a short period in Affecting these compounds and changing their nature in an attempt to get rid of them. Determination of chlorpyrifos breakdown products by using mass scan of Qtrap API $6500+$ LCMS/MS System and Thermo Scientific Q Exactive Focus Orbitrap LC-MS/MS System was investigated. Supported AgV03 NPs, according to the study, could be used in long-term environmental remediation because they can be used in aqueous solutions at room temperature without the need for additional stimulus such as UV light.

\section{Introduction}

The use of pesticides in modern agriculture has become one of the most important necessities to meet the needs of society for food as a result of the continuous increase in the number of the population, and thus millions of tons of pesticides are used annually for this purpose (Rani 2012). Pesticides are among the most widely used chemicals. Despite the importance of using pesticides, they are considered one of the most harmful substances that affect humans, animals, and surface water in particular (McKnight et al. 2015). As a result of applying pesticides in high quantities in the environment, then they may affect surface water and through water storms may lead to a high spread of pollution, which affects aquatic ecosystem (Poulier et al. 2014). Organophosphates are one of pesticide group used to protect plant and increase crop productivity by killing pests and insects. Chlorpyrifos is one of the most popular organophosphate pesticides widely used for controlling pests and diseases for various crops due to its accessibility (Muhamad 2015). Chlorpyrifos (C9H11Cl3NO3PS) 0,0-Diethyl 0-3,5,6-tri chloro pyridinyl phosphorothioate is one of an organophosphate pesticide used to kill a number of pests including insects and worms. It is used on crops, animals, and buildings (Rathod et al. 2017). In the United States, chlorpyrifos was first registered in 1965 for the control of vegetation and soil-borne insects (U.S. EPA 2002). It is one of the most commonly used organophosphate insecticides in agriculture in the United States, and it was one of the most widely used residential insecticides until being phased out (WHO 
2019). The World Health Organization rates chlorpyrifos as moderately harmful to humans. It works on the nervous system of insects by inhibiting acetyl cholinesterase, and its home use was prohibited in the United States in 2001 (Brett Israel 2012). It acts on the nervous system of insects by inhibiting acetyl cholinesterase. Eye watering, increased saliva, sweating, nausea, and headache are all symptoms of Chlorpyrifos poisoning. Muscle spasms or fatigue, vomiting or diarrhoea, and vision impairment are all possible side effects of intermediate exposure. Seizures, unconsciousness, coma, and suffocation from respiratory failure are all symptoms of serious poisoning (Christensen et al. 2014). It is named an overall use pesticide (GUP) and it is enrolled for agribusiness utilizes with 64 yields in the Egypt. An aggregate of 12919 examples of 57 distinct sorts of natural product vegetable and sweet-smelling restorative plants tests were inspected for chlorpyrifos buildups during 2002. It was tracked down that fifteen items address all out 12193 examples of natural products (2634), vegetables (2494) and fragrant therapeutic plants (7065) with rate $94.38 \%$ of absolute analyzed examples have chlorpyrifos buildups (defiled wares). Just $10.16 \%$ of the all-out tainted products (12193 examples) were underneath cutoff of assurance (LOD = $0.02 \mathrm{ppm}), 9.62 \%$ at LOD or all the more anyway $80.21 \%$ of those examples are not polluted with chlorpyrifos buildups. Just $6.33 \%$ of the examples surpassed MRL's of chlorpyrifos buildups contrasting and public, Codex and EU-MRL's. chlorpyrifos buildup were the identified as often as possible in dry celery and cumin tests with rates of $61.54 \%$ and $40.91 \%$ individually and furthermore showed higher infringement rate for similar products $58.97 \%$ and $40.91 \%$. The significant supporters of absolute admission of chlorpyrifos buildup are the root vegetables (81.89\%). Information showed that the all-out dietary admission of chlorpyrifos is to be $0.0045 \mathrm{mg} / \mathrm{kg}$. Body weight/day is lower than ADI $(0.01 \mathrm{mg} / \mathrm{kg}$ body weight) and contributing just $45 \%$ of $A D I$. Consequently dietary openings to chlorpyrifos are as yet not a case for Egyptian customer concern. Because of their far reaching use, organophosphates have caused critical contamination and have amassed in soils, ground water, food harvests, vegetables, and surprisingly in creatures (Coupe et al. 2000; Gül 2005; Selvi et al. 2000), accordingly, it is important to distinguish effective innovations for eliminating them from the climate. Numerous innovations including single strategies and blends of (H2O2) (Ikehata et al. 2006), and chlorine ( $\mathrm{NaOCl}$ ) (Duirk et al. 2005), couldn't successfully corrupt these engineered pesticides. Subsequently, some mix techniques were utilized. The corruption and mineralization of chlorpyrifos was examined utilizing the Fenton reagent (H2O2/Fe2+), and demonstrated that it was successful. Besides, it has been shown that few types of microscopic organisms and growths displayed impressive limit with respect to processing chlorpyrifos in the dirt and fluid media (Eissa et al. 2014; El-Helow et al. 2013; Kulshrestha et al. 2011; Li X et al. 2008; Yadav et al. 2014). Ferrate (VI) was used to study the kinetics of chlorpyrifos, an organophosphorus (OP) compound, and the ability of this iron-based chemical oxidant to remove chlorpyrifos from water and wastewater treatment (Liu et al. 2019). Bimetallic silver/copper $(\mathrm{Ag} / \mathrm{Cu}$ ) nanoparticles was synthesized and then used for water purification application using the toxic pollutant, chlorpyrifos pesticide, as a model (Rosbero et al. 2017). Hexacyanoferrates (MHCFs) of Zn, Cu, Co, and Ni was evaluated in deprivation of selected hazardous pesticides, viz., chlorpyrifos (CP), thiamethoxam (TH), and tebuconazole (TEB). Sharp nanocubes of ZnHCF ( $100 \mathrm{~nm})$, distorted nanocubes of CuHCF $(\sim 100 \mathrm{~nm})$, and nanospheres of CoHCF and NiHCF $(<10 \mathrm{~nm}$ ) were synthesized (Rani et al. 2018). Magnetically separable $\mathrm{Fe}-\mathrm{ZnO}$ nanocomposites were synthesized and then evaluated the photodegradation activity 
of $\mathrm{Fe}-\mathrm{ZnO}$ nanocomposite on an organophosphate pesticide (chlorpyrifos) at different concentration under UV irradiation and analyzed by FT-IR, Raman and UV-Vis spectroscopy (Khan et al. 2018). Ag and Au NPs were used in both approved and unsupported types at room temperature, chlorpyrifos (CP), an organophosphorothioate pesticide, decomposes into 3,5,6-trichloro-2-pyridinol (TCP) and diethyl thiophosphate over. Absorption spectroscopy and electrospray ionisation mass spectrometry were used to identify the degradation products (ESI MS) (Bootharaju et al. 2012). The method presented in this study describes the synthesizing silver vanadate nanoparticles and then using it in degradation of some pesticides. The determination of these compounds were estimated using electrospray positive ionisation (ESI+) by Exion HPLC coupled with Sciex Qtrap API 6500 + LC-MS/MS System. In this study, using mass scan of Qtrap API 6500 + LC-MS/MS System and Thermo Scientific Q Exactive Focus Orbitrap LC-MS/MS System to determine chlorpyrifos breakdown products. The method used before determination is simple and take a short time to reach to these results by using $0.1 \mathrm{~g}$ of silver vanadate nanoparticles.

\section{Experimental Method}

\subsection{Instrumentation and analysis}

(1) LC-MS/MS System, ExionLC AC coupled with Qtrap API 6500 + MS/MS system from AB Sciex, USA.

(2) Chromatographic column, Infinitylab Poroshell 120 EC-C18 $3.0 \times 50 \mathrm{~mm}, 2.7 \mu \mathrm{m}$ particle size (Agilent, USA).

The column temperature was $40^{\circ} \mathrm{C}$ and the injection volume was $2 \mu \mathrm{L}$. The pesticides are separated using Gradient mixing programme of $10 \% 50 \mathrm{mM}$ ammonium acetate in deionised water, with $0.1 \%$ formic acid as eluent $A$ and methanol as eluent $B$ at $300 \mu \mathrm{L} / \mathrm{min}$ flow rate starting by $A$ bottle $60 \%$ for 1 min, changed continuously till $11.5 \mathrm{~min}$ to be $10 \%$ for $0.5 \mathrm{~min}$, changed progressively till $12 \mathrm{~min}$ to be $0 \%$ for 2 min and returned to $60 \%$ from $A$ in min 14 for 2 min to be 16 min complete run time for every one of the 25 pesticides yet for one pesticide the flow rate starting by A bottle $20 \%$, changed steadily till 3 min to be $20 \%$ for $0.5 \mathrm{~min}$ and returned to $20 \%$ from $\mathrm{A}$ in $\mathrm{min} 3.5$ for $1.5 \mathrm{~min}$ to be $5 \mathrm{~min}$ all out run time. The MS/MS investigation was finished by electrospray ionization in the positive ion mode with multiple reactions monitoring (MRM) mode. The following source and gas parameters were applied: temperature, $400^{\circ} \mathrm{C}$; and ion spray voltage, $5500 \mathrm{~V}$.

LC mobile phase stock solution was $50 \mathrm{mM}$ ammonium format solution in methanol/water (1:9), add $1.73 \mathrm{~mL}$ formic acid to $900 \mathrm{~mL}$ water, adjust the $\mathrm{pH}$ to about $3.78 \pm 0.02$ with ammonia solution (33\%) and then add $100 \mathrm{~mL}$ methanol and LC mobile phase was $10 \mathrm{mM}$ ammonium format solution in methanol/water (1:9), dilute $200 \mathrm{~mL}$ of LC mobile phase stock solution with $800 \mathrm{~mL}$ methanol/water (1:9), the $\mathrm{pH}$ should be $4 \pm 0.1$, adjust if necessary.

(3) Q Exactive Focus Orbitrap LC-MS/MS System from Thermo Scientific (USA). 
(4) Chromatographic column, ZORBAX Eclipse Plus C18 $4.6 \times 150$ mm, $5 \mu \mathrm{m}$ particle size (Phenomenex, Torrance, CA, USA).

\subsection{Reagents and materials}

(1) Acetamiprid (98\%), Atrazine (99\%), Azoxystrobin (99\%), Butralin (99\%), Carbendazim (99\%), Carbofuran (98.5\%), Chlorpyrifos (97\%), Chlorpyrifos-methyl (99.5\%), Chlorthiophos (96.5\%), Clethodium (50\%), Clodinafop-propargyl (99\%), Cyhalothrin lambda (98.5\%), Cypermethrin (97\%), Cyprodinil (99\%), Dimethoate (98.5\%), Florasulam (99\%), Malathion (99\%), Methamidophos (98\%), Methomyl (99.5\%), Phenthoate (96.5\%), Piperonyl butoxide (99\%), Pirimiphos-methyl (99\%), Profenofos (99\%), Propargite (99\%) and Tetramethrin (98\%) were purchased from Dr. Ehrenstorfer (Augsburg, Germany).

(2) Methanol (99.9\%) HPLC grade was purchased from J.T. Baker (PA, USA).

(3) Silver nitrate (99.8-100.5\%) HPLC was purchased from sigma aldrich.

(4) Ammonium metavanadate (99\%) HPLC was purchased from sigma aldrich.

(5) Deionised water (<18M_cm resistivity) was performed in the laboratory using a

Millipore (Billerica, MA, USA) MilliQ water purification system.

(6) Ammonia solution (33\%) was purchased from Riedel-de Häen (Seelze, Germany).

(7) Formic acid (98-100\%) was purchased from Riedel-de Häen.

\subsubsection{Standard preparation}

Stock solutions $(1000 \mu \mathrm{g} / \mathrm{mL})$ of each pesticide standard were prepared by dissolving Acetamiprid in toluene, Atrazine in toluene, Azoxystrobin in toluene, Butralin in toluene, Carbendazim in Acetonitrile/Methanol (1:1 v/v), Carbofuran in toluene, Chlorpyrifos in toluene, Chlorpyrifos-methyl in toluene, Clethodium in toluene, Clodinafop-propargyl in toluene, Cyhalothrin lambda in toluene, Cypermethrin in toluene, Cyprodinil in toluene, Dimethoate in toluene, Florasulam in toluene / Acetone (9:1 $\mathrm{v} / \mathrm{v})$, Malathion in toluene, Methamidophos in toluene, methomyl in toluene, Phenthoate in toluene, Piperonyl butoxide in toluene, Pirimiphos-methyl in toluene, Profenofos in toluene, Propargite in toluene and Tetramethrin in toluene. All stock solutions were prepared and kept at $-20 \pm 2{ }^{\circ} \mathrm{C}$. Working mixture standard solution of the studied pesticides $(20 \mu \mathrm{g} / \mathrm{mL}$, each) and calibration mixtures of concentration levels $0.01,0.05$ and $0.5 \mu \mathrm{g} / \mathrm{mL}$ were prepared by diluting suitable aliquot of the stock solutions with methanol stored at $4 \pm 2{ }^{\circ} \mathrm{C}$.

\subsection{Extraction procedure}

\subsubsection{Synthesis of silver vanadate AgVO3 nanoparticles}


The procedure involves 0.01 mole of ammonium vanadate was dissolved in $20 \mathrm{ml}$ of deionized water and 0.01 mole of silver nitrate was dissolved in $20 \mathrm{ml}$ of deionized water. The silver nitrate solution was added gradually to the ammonium vanadate solution with ultrasonic stirring at room temperature. The $\mathrm{PH}$ of colored suspension was adjusted to near 7 using ammonia solution and ultrasonic stirring continued to 3 hour. The obtained suspension was then transferred into Teflon-lined vessel and placed in a microwave oven operating at $2.5 \mathrm{GHz}$ and maximum power of $650 \mathrm{~W}$ at $160^{\circ} \mathrm{C}$ for 2 hour. The resulting precipitate was filtered and washed several times with deionized water and ethanol using centrifuge for 5 minute at $13000 \mathrm{rpm}$ at each time. After washing, the precipitate was dried at $80^{\circ} \mathrm{C}$ for 24 hour. At long last the subsequent powder was granulated well to utilize it.

\subsubsection{Degradation of pesticides using AgVO3 nanoparticles}

The procedure involves $0.1 \mathrm{~g}$ of AgVO3 nanoparticles were added to $100 \mathrm{ml}$ of mixture of pesticides 0.5 $\mu \mathrm{g} / \mathrm{mL}$ dissolved in in methanol/water $(1: 1 \mathrm{v} / \mathrm{v})$ using a magnetic stirring to reach equilibrium. AT chosen time intervals, sample aliquots were collected, centrifuged for $5 \mathrm{~min}$ at $4000 \mathrm{rpm}$ (3430 rcf) and filtered using syringe filters $(0.45 \mu \mathrm{m})$ and then transferred to Polypropylene (PP) vial. Finally, the filtrates were further analyzed by LC-MS/MS. The previous steps were repeated again with each pesticide that affected individually. This experiment was conducted repeated several times in dark and visible light using UV lamp at $365 \mathrm{~nm}$ and without it at room temperature.

\section{Result And Discussion}

A chemical remediation study was evaluated to reducing chlorpyrifos risk from irrigation water. Some chemicals used as catalyst were prepared in Nano-particle phase and were tested in vitro to check their ability to cracking out tested pesticide. An analytical study was used to evaluate catalysts efficiency and the environmental fats for chlorpyrifos using liquid chromatography tandem mass spectrometry. In previews studies the use of gas chromatography coupled with single mass were used to checks compounds with and without a chemical fragmentation test, Also infra-red and NMR determination were used to support compound fat structures. In this investigation a tandem mass was used for the determination of chlorpyrifos breakdown products by using mass scan before multiple reactions monitoring mechanism as a finger prints for the chemical reaction products and also to have relationship between the main compound and its founded products.

Twenty five pesticides were tested by silver vanadate nanoparticles in visible light at room temperature for 24 hours, only 3 pesticides (chlorpyrifos, dimethoat, malation) were affected till be displayed from solution test, Its clearly they relationships to each other as a organophosphate classification which led us to physicochemical theory about the reaction of silver vanadate nanoparticle to breakdown persisted pesticides in environment specially in irrigation water.

Table 1 showed dramatically dissipation in 15 min for chlorpyrifos and dimethoat when malation was more resist for more $30 \mathrm{~min}$. 
An analytical study was done to investigate chlorpyrifos breakdown fragments using tandem mass spectrometry ( $Q$ trap) and hybrid orbitap technique for mass scanning and exact mass determination, Chlorpyrifos and its breakdown derivatives were scanned and determined as a way to investigates bonds breaking pathway.

Figure 1 showed the mass scan of chlorpyrifos by first mass filter including the isotopic distribution of compound due to the present of 3 chlorine atoms, After scanning 350 Dalton was used as mass separation for parent chlorpyrifos in Q1 before special fragmentation in collision cell for two fragments (274 and 198), then Q3 was used as linear ion trap for filtering each fragment before second fragmentation and scanning to found up third mass part from analyte (MS/MS/MS).

Figures 2 and 3 showed two different fragments comes from tow fragments from chlorpyrifos $(350,274,144$ and $350,198,107)$ as a triple mass finger print for tested analyte. The present of 144 Dalton fragment from 198 parent ion was due to the loss of $\mathrm{H} 2 \mathrm{O}+\mathrm{Cl}$ when the other fragments 107 was due $\mathrm{CO} 2+\mathrm{Cl}+\mathrm{HCN}$ losses [24]. From the other hand, multiple reactions monitoring mechanism was used to determines all founded compounds in the solution after one day from chlorpyrifos silver vanadate nanoparticles reaction. Figure 4 showed the full scan results found in solution before optimizing best energy and mass unit voltage to found up fragments for these compounds derivatives.

Figures 5 and 6 showed the products of two chosen breakdown fragment, the first one was 174 which broken to 5 detected fragment under instrumental fragmentation and the other was 198 gives 2 fragments one of them was found as third products to main chlorpyrifos, this result led us to similar found in between chemical and instrumental degradation. The present of 198, 199.8 and 202 masses was due to $35 \mathrm{Cl} 3,35 \mathrm{Cl} 237 \mathrm{Cl}$, and $35 \mathrm{Cl} 37 \mathrm{Cl} 2$ isotopomers of TCP $(3,5,6$-trichloro-2- pyridinol) respectively.

Mass determination with high resolution was studied using hybrid orbitrap mass spectrometry. A high accuracy scan ranged between 85 to 355 Dalton was done for tested solution after Nano reaction as shown in Fig. 7; instrumental method was adjusted for both scanning and special fragmentation for any founded masses between target ranges. Four masses were found in confirming with previews test (Qtrap$6500+)$ : 283.27, 274.27, 197.92 and 169.06.

As showed in Figs. (8-12) the fragments ions with high abundance produced by founded masses after Nano reaction were found $113,106.945,108.9423,209.1536$ and 96.0704 respectively which were supported by the triple quadrupole mass instrument and led us to have good idea about the breakdown path way of chlorpyrifos pesticide after remediation reaction with Nano silver vanadate.

This data was in agreement with that found before, the products produced by silver nitrate nanoparticles reaction in 48 hours led to protonated TCP 198 Dalton which produced 144 and 107 Dalton fragments when using 3200 QTrap MS/MS instrument (Bootharaju et al. 2012). Also an accurate mass of 197.9235 was found when scanning chlorpyrifos after Fe oxidation using Triple TOF 5600 mass spectrometry instrument (Liu et al. 2019). From the other hand, the present of 107 as a fragment product from both 
parents 298 and 274 can explain a possible structure of 274 which was supported in the shown spectrum of TOF triple mass for chlorpyrifos fragmentation (Liu et al. 2019). The use of silver/copper nanoparticles as a catalysts for chlorpyrifos degradation led to TCP and DETP (Diethyl thiophosphate) (Rosbero et al. 2017).

Possible structures for chorpyrifos breakdowns were showed in Figs. (8-12) of orbitrap MS2 scanning and fragmentation can explained the possible pathway of chlorpyrifos when using Nano-silver vanadate in aqueous solution as in vitro examination for this pesticide remediation.

Possible structures for chlorpyrifos breakdowns were showed in Fig. 13 and supported by all previews data that the fragmentations 282 and 198 could be found due to the loss of 2 chlorine atoms in a substitution reaction in chlorpyrifos to get 282 mass and by breaking the single bond between oxygen and phosphorus to get 198 mass, this theory can be supported by the isotopic distribution showed in this scan Fig. 13 and also can explained the possible pathway of chloropyrifos when using silver vanadate nanoparticles in aqueous solution as in vitro examination for this pesticide remediation.

\section{Conclusion}

The present study used a mass scan of the Qtrap API 6500 + LC-MS/MS System and the Thermo Scientific Q Exactive Focus Orbitrap LC-MS/MS System to establish a rapid and simple method for determining chlorpyrifos breakdown products after reaction with synthesized silver vanadate nanoparticles (AgV03). The addition of $0.1 \mathrm{~g}$ of silver vanadate nanoparticles (AgVO3) was enough to decompose $0.5 \mathrm{mg} / \mathrm{kg}$ of chlorpyrifos, dimethoate and malathion in $100 \mathrm{ml}$ water/ methanol $(1: 1 \mathrm{v} / \mathrm{v})$ and breakdown to their products at room temperature in 15 minutes, which was regarded a short period. The addition of $0.1 \mathrm{~g}$ of silver vanadate nanoparticles (AgVO3) were added to different ranges of concentrations of these chemicals up to $5 \mathrm{mg} / \mathrm{kg}$ and gave the same results. These chemicals were identified using an electrospray positive ionisation (ESI+) by an Exion HPLC paired with a Sciex Qtrap API $6500+$ LC-MS/MS system. To determine chlorpyrifos breakdown products, the mass scans of the Qtrap API 6500 + LC-MS/MS System and the Thermo Scientific Q Exactive Focus Orbitrap LC-MS/MS System were employed. The use of a UV lamp, as well as conducting the experiment in the dark, yielded negative findings. When the experiment was carried out in visible light at room temperature, good findings were obtained. The breakdown products of chlorpyrifos were obtained in a short amount of time, demonstrating the clear action of silver vanadate nanoparticles. The synthesized silver vanadate nanoparticles (AgVO3) may be used as a packed filter to avoid its spread in water and it may be increased its efficiency by adding an element as a Nano composite material. The fact that degradation occurs at room temperature and on supported NPs, on the other hand, is significant since it allows such materials to be used for water filtration. In such cases, a post degradation phase using adsorbents such as activated carbon is required to remove the degradation products.

\section{Declarations}




\section{Acknowledgements}

The authors acknowledge the assistance of the Central Laboratory of Residue Analysis of Pecticides and Heavy Metals in Foods, which was awarded an international Accreditation Certificate in all analyses by the Finnish Branch (FINAS) of the European Accreditation Center for Laboratories (EAL) based on ISO 45001, which was updated in 1999 to ISO/IEC 17025.

\section{Authors' contributions}

All authors contributed to the study conception and design. Material preparation, data collection and analysis were performed by Islam R. Ghoniem, Ashraf M. Elmarsafy, Mohamed A. Amer, Hassan A. ElGammal, Mostafa A. Ahmed and Mohamed M. Abo Aly. The first draft of the manuscript was written by Islam R. Ghoniem and all authors commented on previous versions of the manuscript. All authors read and approved the final manuscript.

\section{Funding}

Not applicable. This study was supported by Central Laboratory of Residue Analysis of Pecticides and Heavy Metals in Foods, which the authors are working in it.

\section{Data availability}

The datasets used and/or analyzed during the current study are available from the corresponding author on reasonable request.

Ethics approval and consent to participate Not applicable

Consent for publication Not applicable

Competing interests The authors declare no competing interests.

\section{References}

1. Bootharaju MS, Pradeep T (2012) Understanding the degradation pathway of the pesticide, chlorpyrifos by noble metal nanoparticles. Langmuir 28:2671-2679. https://doi.org/10.1021/la2050515

2. Brett Israel (2012) Common Insecticide May Harm Boys' Brains More Than Girls. Scientific American

3. Christensen K, Harper B, Luukinen B, Buhl K, Stone D (2014) Chlorpyrifos Technical Fact Sheet. National Pesticide Information Center

4. Coupe RH, Manning MA, Foreman WT, Goolsby D, Majewski M.S (2000) Occurrence of pesticides in rain and air in urban and agricultural areas of Mississippi, April-September 1995. Sci Total Environ 248:227-240. https://doi.org/10.1016/S0048-9697(99)00545-8 
5. Duirk SE, Collette TW (2005) Organophosphate pesticide degradation under drinking water treatment conditions. USEPA, EPA/600/R-05/103

6. Eissa FI, Mahmoud HA, Massoud ON, et al (2014) Biodegradation of chlorpyrifos by microbial strains isolated from agricultural wastewater. J Am Sci 10:98-108

7. El-Helow ER, Badawy MEI, Mabrouk MEM, et al (2013) Biodegradation of chlorpyrifos by a newly isolated bacillus subtilis strain, Y242. Bioremediat J 17:113-123.

https://doi.org/10.1080/10889868.2013.786019

8. Gül A (2005) Investigation of acute toxicity of chlorpyrifos-methyl on Nile tilapia (Oreochromis niloticus L.) larvae. Chemosphere 59:163-166. https://doi.org/10.1016/j.chemosphere.2004.10.065

9. Ikehata K, El-Din MG (2006) Aqueous pesticide degradation by hydrogen peroxide/ultraviolet irradiation and Fenton-type advanced oxidation processes: A review. J Environ Eng Sci 5:81-135. https://doi.org/10.1139/s05-046

10. Khan SH, Pathak B, Fulekar MH (2018) Synthesis, characterization and photocatalytic degradation of chlorpyrifos by novel Fe: ZnO nanocomposite material. Nanotechnol Environ Eng 3:1-14. https://doi.org/10.1007/s41204-018-0041-3

11. Kulshrestha G, Kumari A (2011) Fungal degradation of chlorpyrifos by Acremonium sp. strain (GFRC1) isolated from a laboratory-enriched red agricultural soil. Biol Fertil Soils 47:219-225. https://doi.org/10.1007/s00374-010-0505-5

12. Liu H, Chen J, Wu N, et al (2019) Oxidative degradation of chlorpyrifos using ferrate(VI): Kinetics and reaction mechanism. Ecotoxicol Environ Saf 170:259-266.

https://doi.org/10.1016/j.ecoenv.2018.11.132

13. Li X, Jiang J, Gu L, et al (2008) Diversity of chlorpyrifos-degrading bacteria isolated from chlorpyrifos-contaminated samples. Int Biodeterior Biodegrad 62:331-335. https://doi.org/10.1016/j.ibiod.2008.03.001

14. McKnight US, Rasmussen JJ, Kronvang B, et al (2015) Sources, occurrence and predicted aquatic impact of legacy and contemporary pesticides in streams. Environ Pollut 200:64-76. https://doi.org/10.1016/j.envpol.2015.02.015

15. Muhamad SG (2010) Kinetic studies of catalytic photodegradation of chlorpyrifos insecticide in various natural waters. Arab J Chem 3:127-133. https://doi.org/10.1016/j.arabjc.2010.02.009

16. Poulier G, Lissalde S, Charriau A, et al (2014) Can POCIS be used in Water Framework Directive (2000/60/EC) monitoring networks? A study focusing on pesticides in a French agricultural watershed. Sci Total Environ 497-498:282-292. https://doi.org/10.1016/j.scitotenv.2014.08.001

17. Rani M (2012) Studies on decay profiles of quinalphos and thiram pesticides. Ph.D Thesis, Indian Institute of Technology Roorkee, Roorkee, Uttarakhand, India, Chapter 1, 5

18. Rani M, Shanker U (2018) Removal of chlorpyrifos, thiamethoxam, and tebuconazole from water using green synthesized metal hexacyanoferrate nanoparticles. Environ Sci Pollut Res 25:1087810893. https://doi.org/10.1007/s11356-018-1346-2 
19. Rathod AL, Garg RK (2017) Chlorpyrifos poisoning and its implications in human fatal cases: A forensic perspective with reference to Indian scenario. J Forensic Leg Med 47:29-34. https://doi.org/10.1016/j.jflm.2017.02.003

20. Rosbero TMS, Camacho DH (2017) Green preparation and characterization of tentacle-like silver/copper nanoparticles for catalytic degradation of toxic chlorpyrifos in water. J Environ Chem Eng 5:2524-2532. https://doi.org/10.1016/j.jece.2017.05.009

21. Selvi M, Sarikaya R, Erkoç F, Koçak O (2005) Investigation of acute toxicity of chlorpyrifos-methyl on guppy Poecilia reticulata. Chemosphere 60:93-96.

https://doi.org/10.1016/j.chemosphere.2004.11.093

22. States U, Substances T (2002) Interim Reregistration Eligibility Decision for Chlorpyrifos

23. The WHO (2019) Recommended Classification of Pesticides by Hazard and Guidelines to Classification

24. Yadav M, Srivastva N, Singh RS, et al (2014) Biodegradation of chlorpyrifos by Pseudomonas sp. in a continuous packed bed bioreactor. Bioresour Technol 165:265-269. https://doi.org/10.1016/j.biortech.2014.01.098

\section{Tables}

Table 1

Tested pesticides breakdown and dissipation rate after nanoparticles fragmentation reaction.

\begin{tabular}{|c|c|c|c|c|c|c|c|c|c|}
\hline \multirow[t]{2}{*}{ B.D. Reagent (AgVO3) } & \multicolumn{3}{|c|}{ Chlorpyrifos $(0.5 \mathrm{mg} / \mathrm{kg})$} & \multicolumn{3}{|c|}{ Dimethoate $(0.5 \mathrm{mg} / \mathrm{kg})$} & \multicolumn{3}{|c|}{ Malathion $(0.5 \mathrm{mg} / \mathrm{kg})$} \\
\hline & P. area & A. Conc. & D. Rate & P. area & A. Conc. & D. Rate & P. area & A. Conc. & D. Rate \\
\hline before adding & $1.26 \mathrm{E}+08$ & 0.499 & $0 \%$ & $7.67 \mathrm{E}+07$ & 0.495 & $0 \%$ & $1.44 \mathrm{E}+08$ & 0.499 & $0 \%$ \\
\hline after $1 \mathrm{~min}$ & $1.03 \mathrm{E}+08$ & 0.404 & $19.0 \%$ & $6.94 \mathrm{E}+07$ & 0.448 & $9.5 \%$ & $1.29 \mathrm{E}+08$ & 0.446 & $10.6 \%$ \\
\hline after $5 \mathrm{~min}$ & $2.19 \mathrm{E}+07$ & 0.0865 & $82.7 \%$ & $4.49 \mathrm{E}+07$ & 0.29 & $41.4 \%$ & $9.06 \mathrm{E}+07$ & 0.313 & $37.3 \%$ \\
\hline after $15 \mathrm{~min}$ & $7.65 \mathrm{E}+06$ & 0.0302 & $93.9 \%$ & $8.30 \mathrm{E}+06$ & 0.0536 & $89.2 \%$ & $4.43 \mathrm{E}+07$ & 0.153 & $69.3 \%$ \\
\hline after $30 \mathrm{~min}$ & & N.D & & $4.37 \mathrm{E}+05$ & 0.00282 & $99.4 \%$ & $2.20 \mathrm{E}+07$ & 0.0762 & $84.7 \%$ \\
\hline after $45 \mathrm{~min}$ & & & & & N.D & & $1.44 \mathrm{E}+07$ & 0.0498 & $90.0 \%$ \\
\hline after $60 \mathrm{~min}$ & & & & & & & & N.D & \\
\hline
\end{tabular}

B. D.: breakdown, P: peak, A. Conc.: analyte concentration and D: dissipation

\section{Figures}




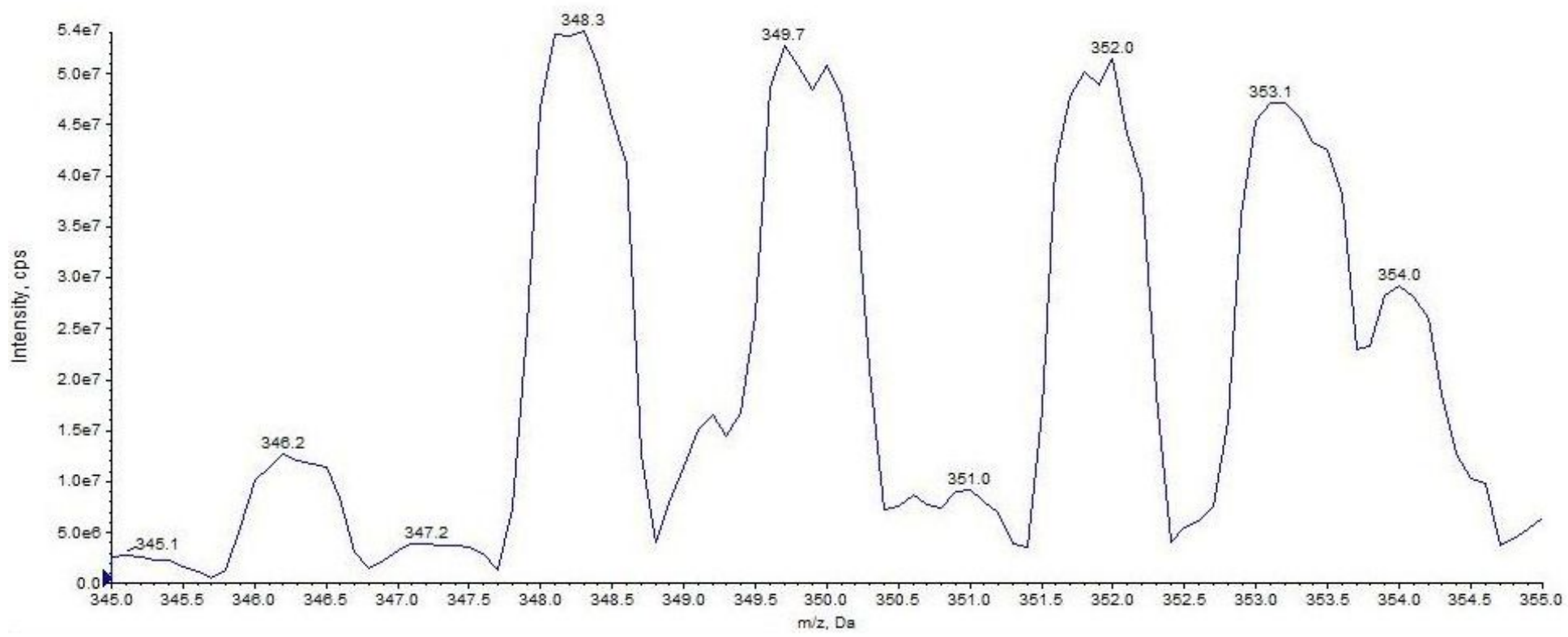

Figure 1

Choropyrifos $(0.1 \mathrm{mg} / \mathrm{kg})$ scanning using Q1 of tandem mass.

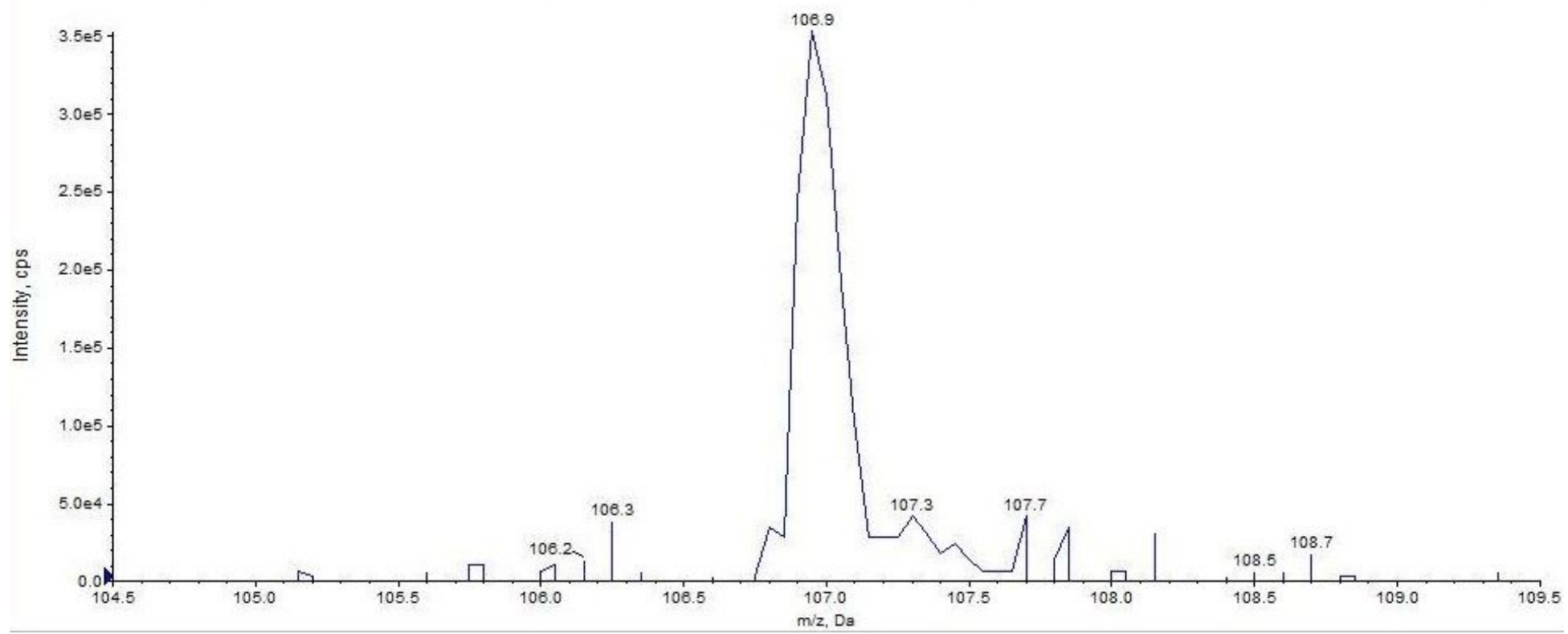

Figure 2

Chlorpyrifos triple followed mass filters using Q trap mechanism $(350,198,107)$. 


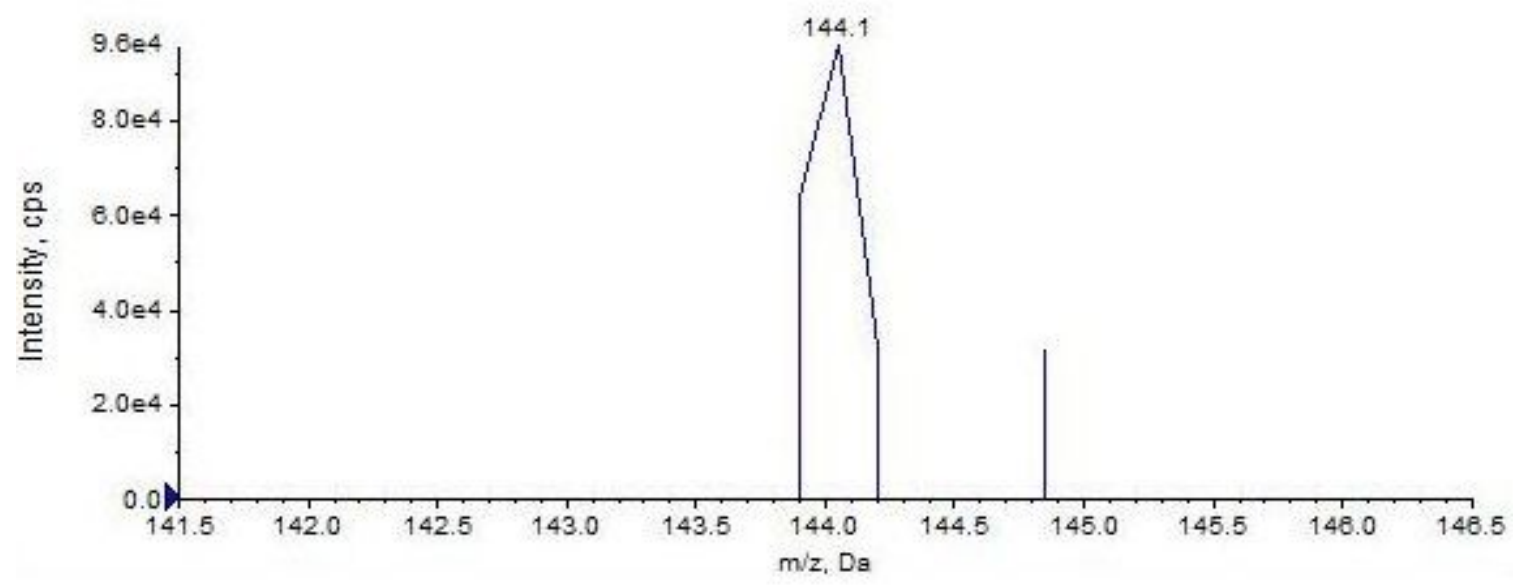

Figure 3

Chlorpyrifos triple followed mass filters using Q trap mechanism $(350,274,144)$.

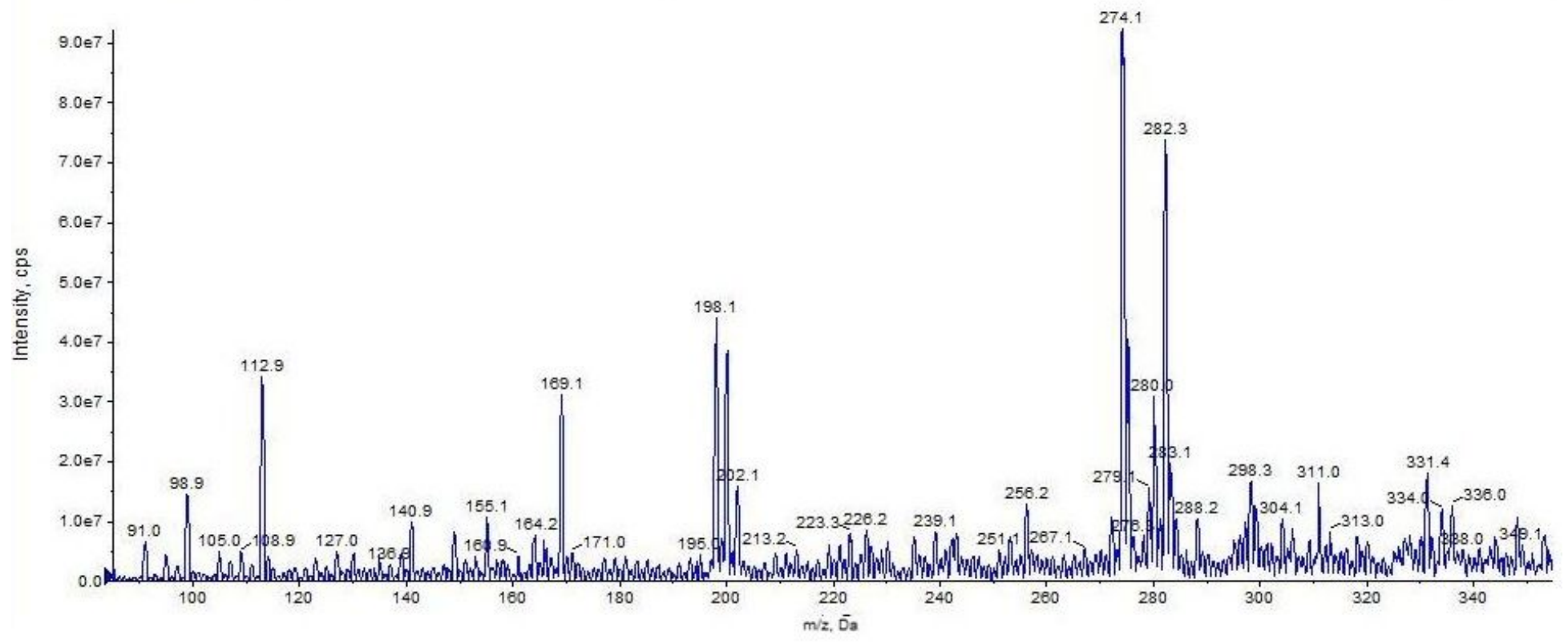

Figure 4

Full scan spectrum for chlorpyrifos derivatives using first quadrupoles Q1. 


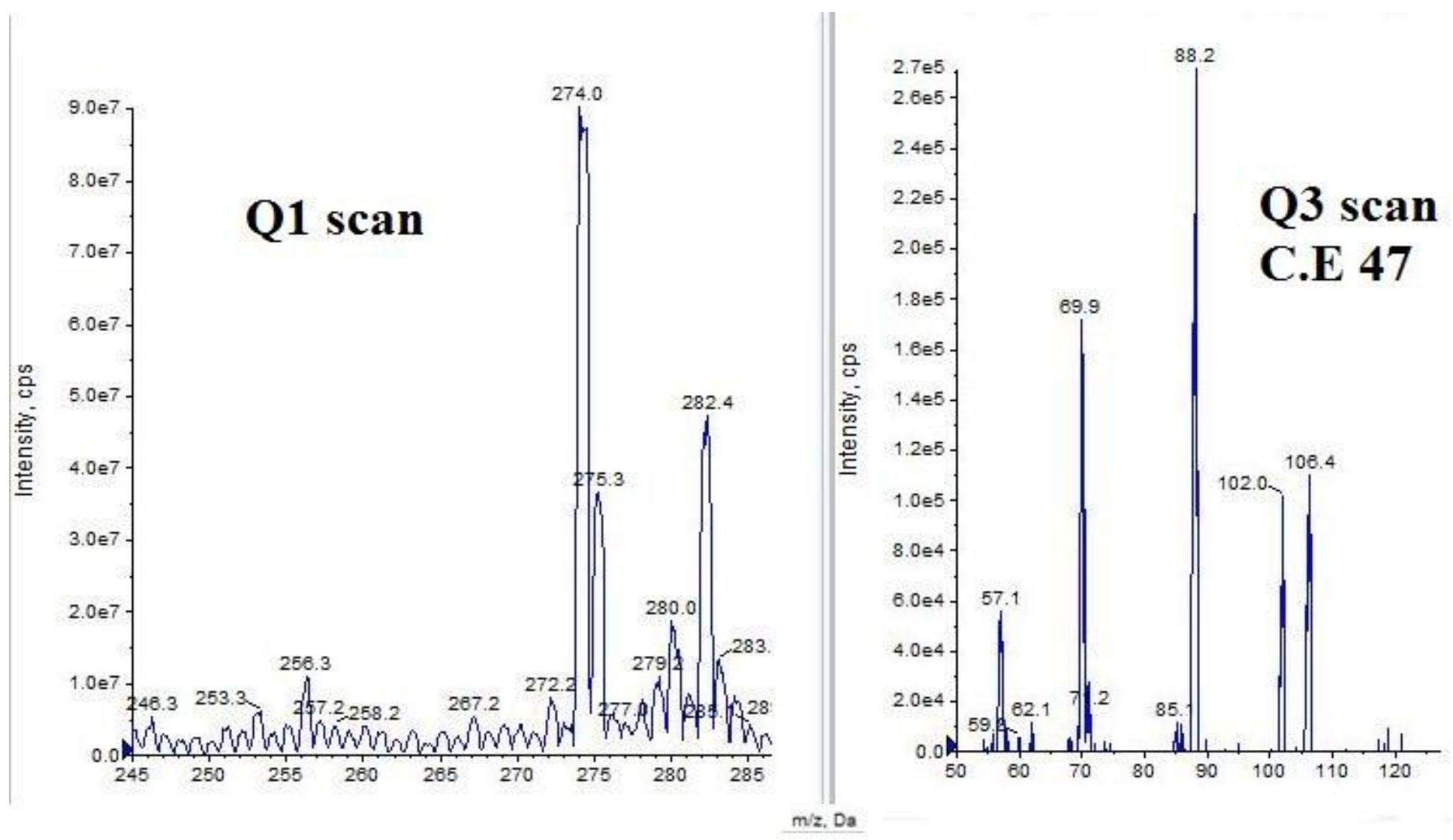

Figure 5

MRM optimization for 274 parent with C.E 47 and fragments scan.

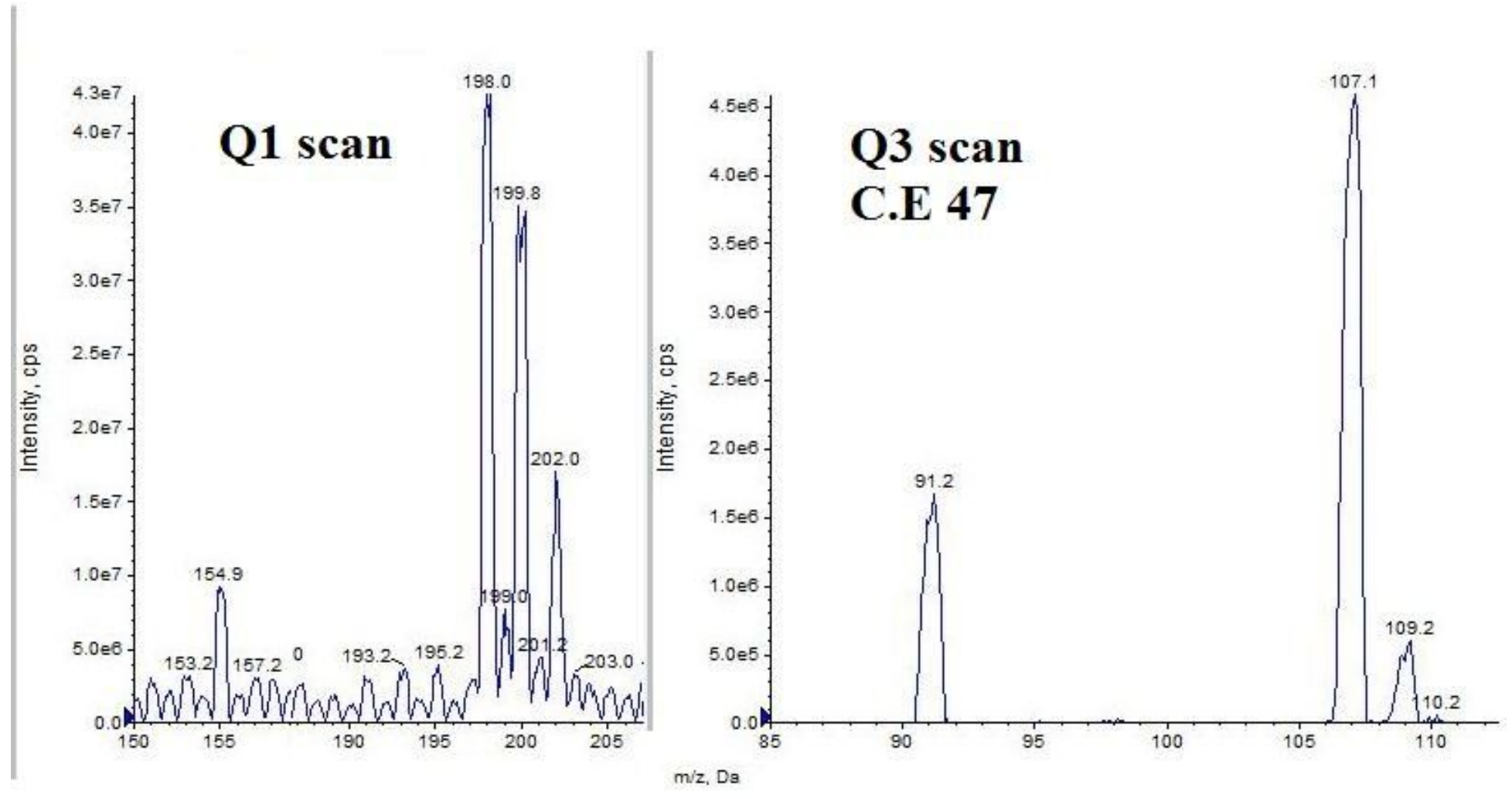

Figure 6 
MRM optimization for 198 parent with C.E 47 and fragments scan.

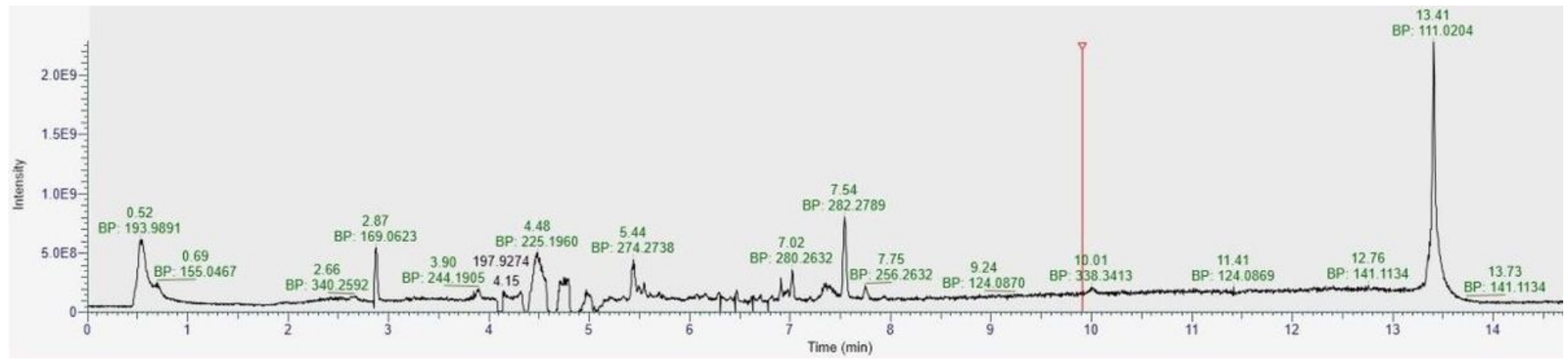

Figure 7

Total ions chromatogram for all residues after nanoparticles reaction using Orbitrap scanning mode.

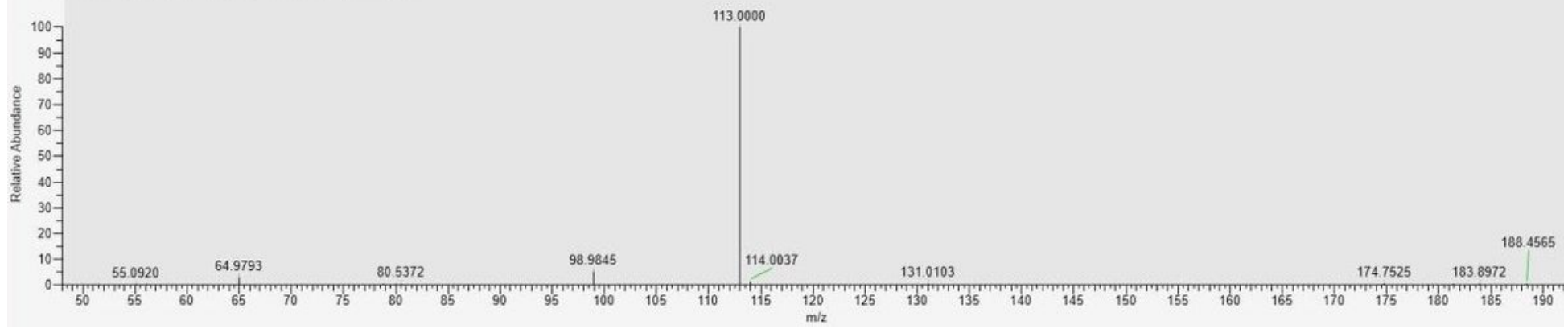

\section{Figure 8}

Big abundance ion after fragmentation of $169 \mathrm{Da}$ ion using Orbitap MS2.

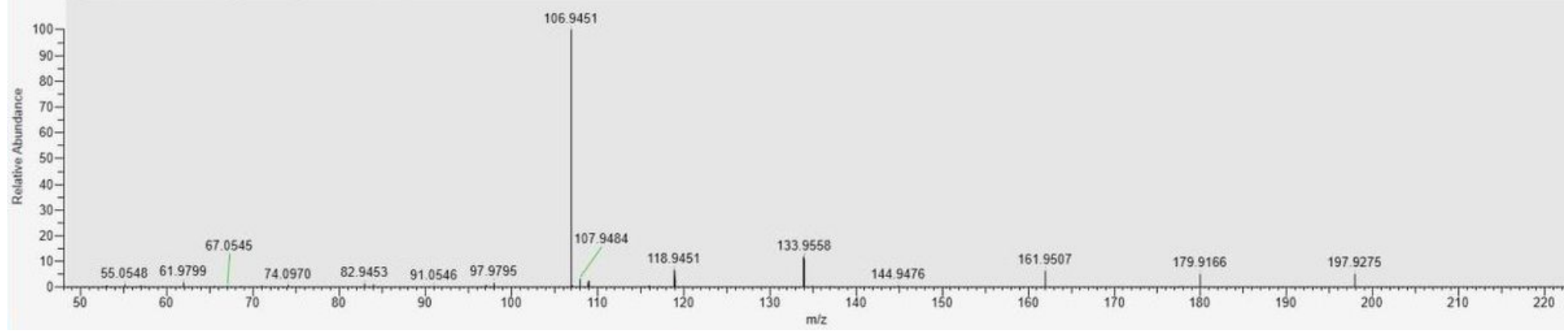

\section{Figure 9}

Big abundance ion after fragmentation of 197.9 Da ion using Orbitap MS2.

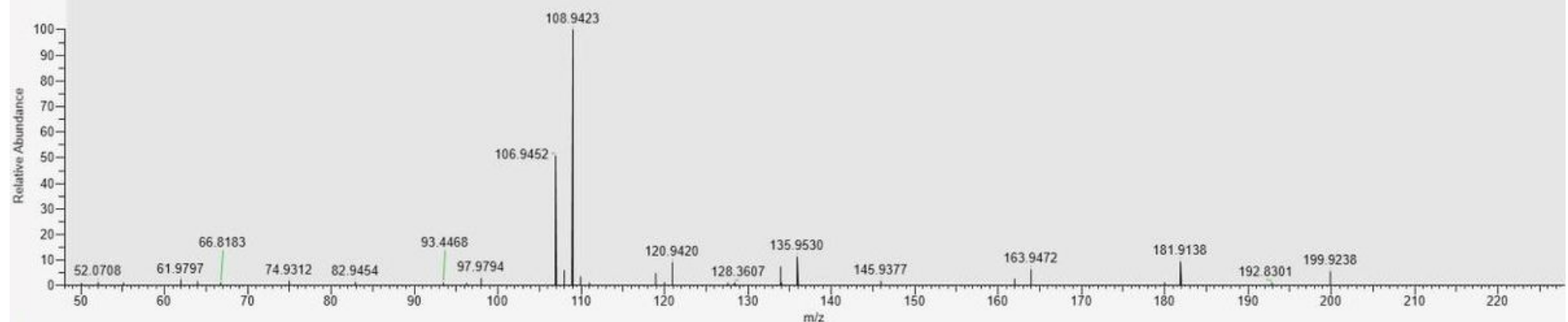


Figure 10

Big abundance ion after fragmentation of 199.9 Da ion using Orbitap MS2.

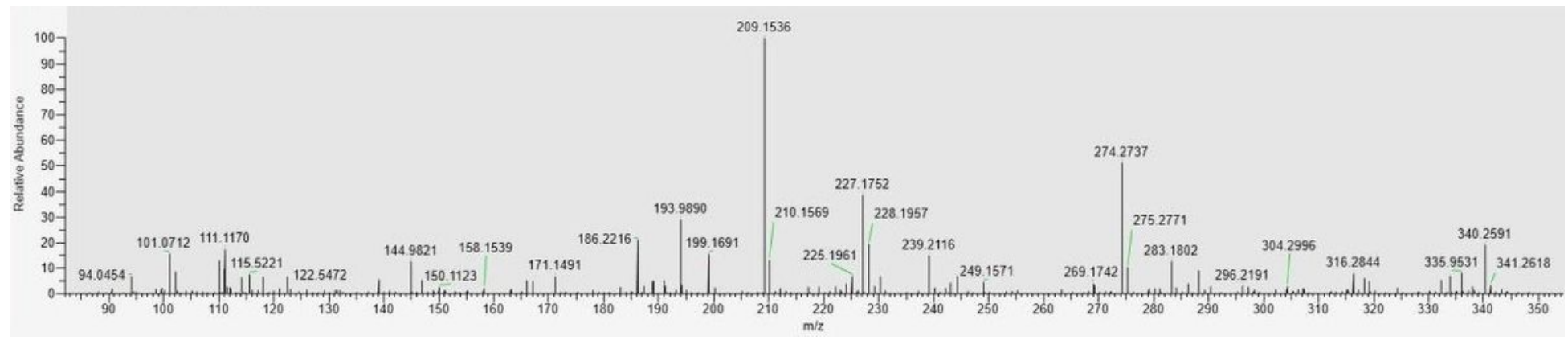

\section{Figure 11}

Big abundance and parent ions after fragmentation of $174 \mathrm{Da}$ ion using Orbitap san and MS2.

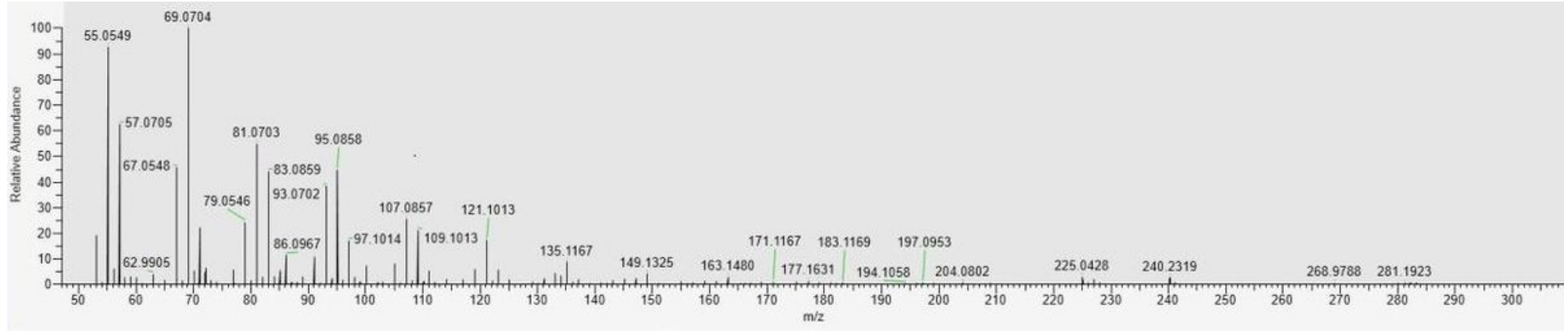

Figure 12

All fragmentations ions of 282.27 Da ion using Orbitap MS2.

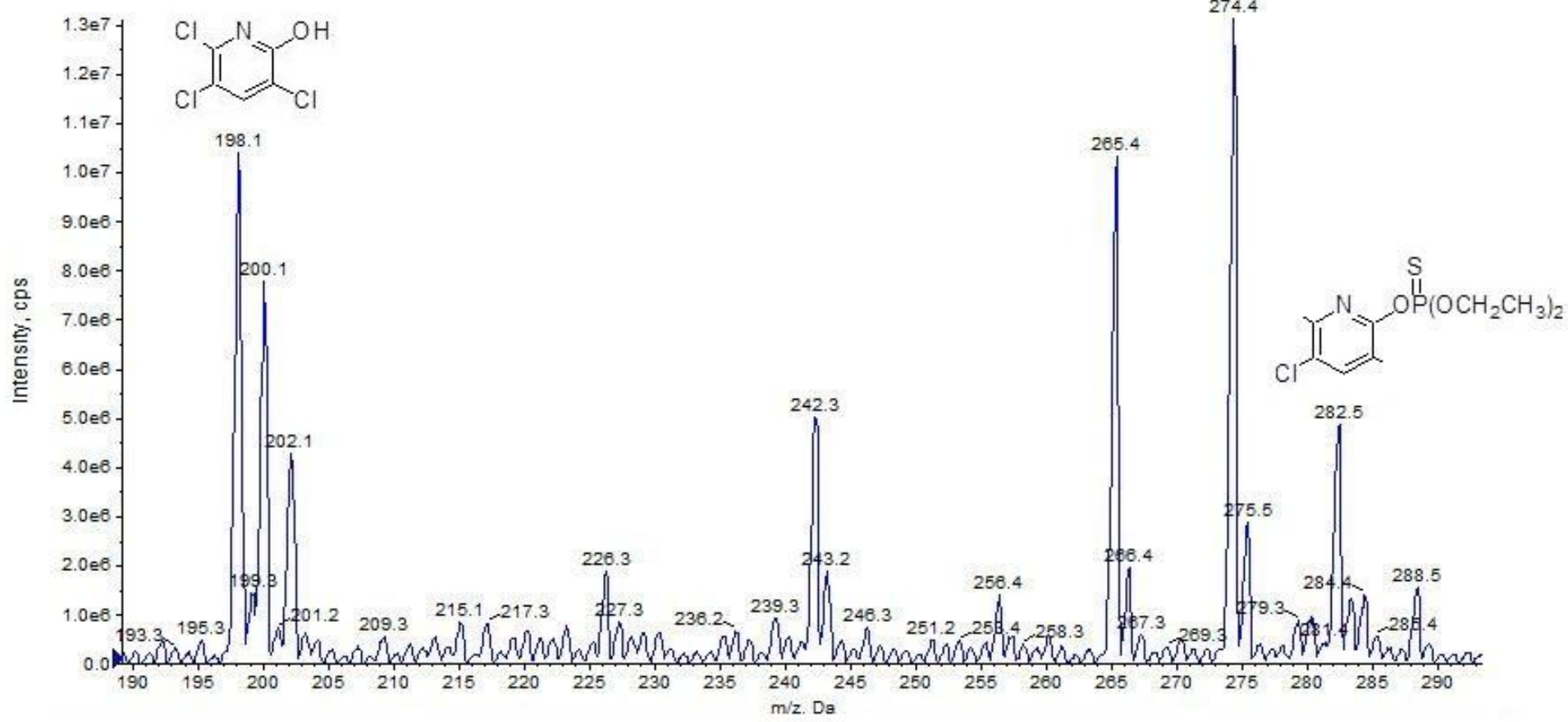


Figure 13

Q1 scanning from the most high abundance fragments produced after reaction. 